\title{
AMENDMENTS
}

\section{Author Correction: Affinity-based capture and identification of protein effectors of the growth regulator $\mathrm{ppGpp}$}

Boyuan Wang, Peng Dai (D), David Ding, Amanda Del Rosario, Robert A. Grant, Bradley L. Pentelute and Michael T. Laub (D)

Correction to: https://doi.org/10.1038/s41589-018-0183-4, published online 17 December 2018.

In the version of the article originally published, two sets of labels on the $x$ axis of the graph in Fig. 5b were in reverse order. In the 'PurF' row, the locations of 'N48A' and 'R45A' should be switched, and in the row below those of ' 4.1 ' and the minus sign should be switched. Shown below are the original and corrected versions of Fig. 5b. The error has been corrected in the HTML and PDF versions of the article.
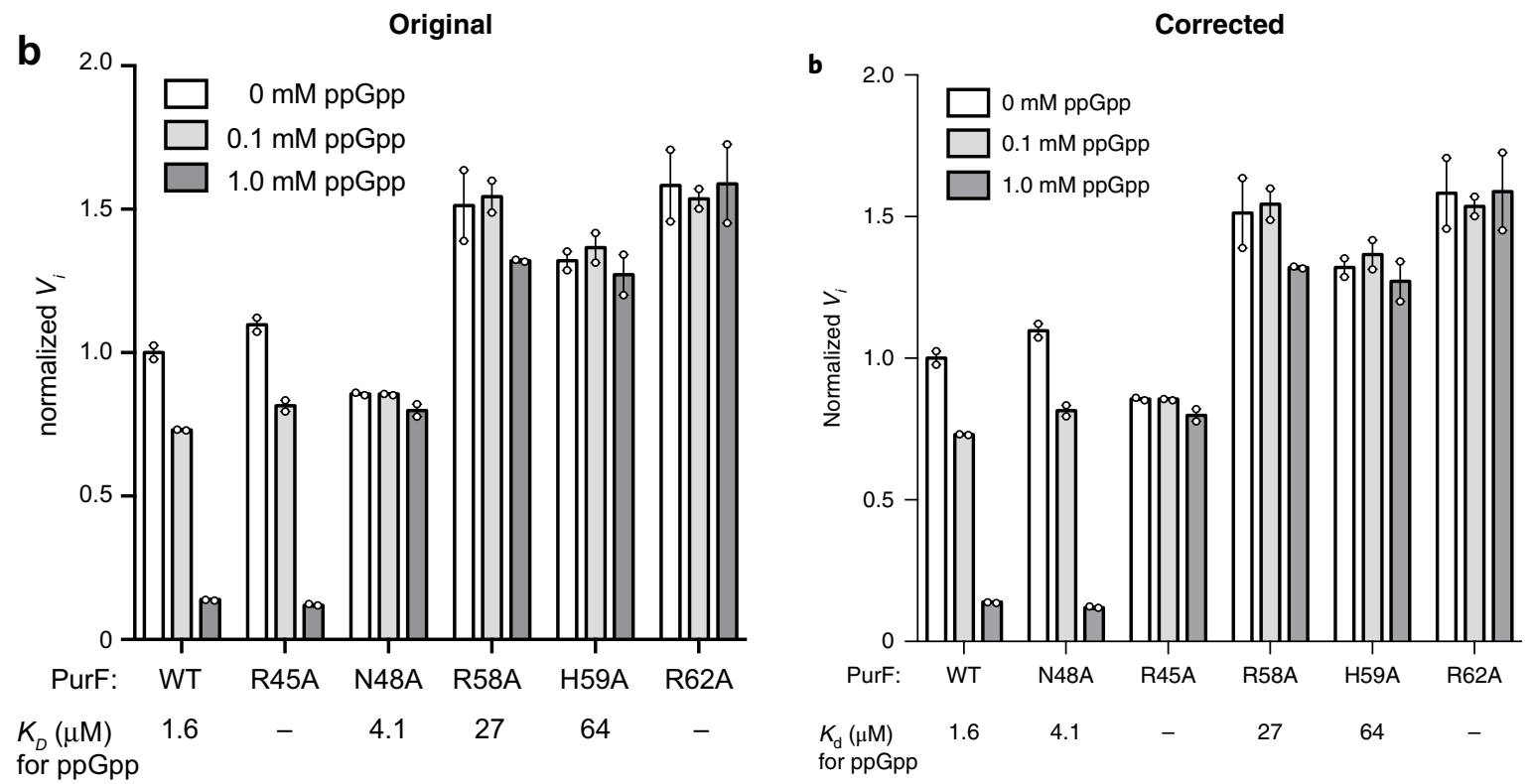

Fig. $\mathbf{5}$ | Original and Corrected. 